\title{
Interaction between Amyloid- $\beta$ Pathology and Cortical Functional Columnar Organization
}

\author{
Shlomit Beker, ${ }^{1}$ Vered Kellner, ${ }^{1}$ Lucia Kerti, ${ }^{1,3}$ and Edward A. Stern ${ }^{1,2}$ \\ ${ }^{1}$ Brain Research Center, Faculty of Life Sciences, Bar-Ilan University, Ramat Gan, 52900 Israel, ${ }^{2}$ MassGeneral Institute of Neurodegenerative Disease, \\ Department of Neurology, Massachusetts General Hospital and Harvard Medical School, Boston, Massachusetts 02129, ${ }^{3}$ Bernstein Center Freiburg, \\ Neurobiology and Biophysics, Faculty of Biology, University of Freiburg, 79104 Freiburg, Germany
}

Amyloid- $\beta$ plaques are one of the major neuropathological features in Alzheimer's disease (AD). Plaques are found in the extracellular space of telencephalic structures, and have been shown to disrupt neuronal connectivity. Since the disruption of connectivity may underlie a number of the symptoms of $\mathrm{AD}$, understanding the distribution of plaques in the neuropil in relation to the connectivity pattern of the neuronal network is crucial. We measured the distribution and clustering patterns of plaques in the vibrissae-receptive primary sensory cortex (barrel cortex), in which the cortical columnar structure is anatomically demarcated by boundaries in Layer IV. We found that the plaques are not distributed randomly with respect to the barrel structures in Layer IV; rather, they are more concentrated in the septal areas than in the barrels. This difference was not preserved in the supragranular extensions of the functional columns. When comparing the degree of clustering of plaques between primary sensory cortices, we found that the degree of plaques clustering is significantly higher in somatosensory cortex than in visual cortex, and these differences are preserved in Layers II/III. The degree of areal discontinuity is therefore correlated with the patterns of neuropathological deposits. The discontinuous anatomical structure of this area allows us to make predictions about the functional effects of plaques on specific patterns of computational disruption in the AD brain.

\section{Introduction}

The amyloid hypothesis is one of the central components of current theories of Alzheimer's disease (AD). Amyloid- $\beta$ $(\mathrm{A} \beta)$, the major constituent of senile plaques in $\mathrm{AD}$, appears to be a critical component of the pathophysiological cascade that leads to dementia (Hsiao et al., 1996). The deposition of A $\beta$ precedes and induces at least some of the neuronal abnormalities that underlie dementia in AD (Selkoe, 2001). Axonal, dendritic, and synaptic defects are each associated with $\mathrm{A} \beta$ in different experimental systems (Mehraein et al., 1975; Selkoe, 2002; Spires and Hyman, 2004; Spires et al., 2005; Palop et al., 2006; Spires-Jones et al., 2007) Spine loss occurs prominently near plaques (Tsai et al., 2004; Spires et al., 2005), and plaques reduced numbers of synapses (Mattson, 2004).

The specific mechanisms by which plaques disrupt neural function remain uncertain (Hyman, 2006). One possible influence of plaques on nerve fibers is that they cause a morphological and functional change in the nerve cells, which in turn influence the connectivity, and hence the functionality of the network. Plaque aggregation is associated with significant neuritic path distortion, and the curvature of neuronal processes is increased in

Received May 20, 2012; accepted June 13, 2012.

Author contributions: E.A.S. designed research; S.B., V.K., and L.K. performed research; S.B., V.K., and L.K. analyzed data; S.B. and E.A.S. wrote the paper.

This work was supported by the National Institute on Aging (Grant AG024238) and the Legacy Heritage BioMedical Program of the Israel Science Foundation (Grant 688/10).

Correspondence should be addressed to Dr. Edward A. Stern, Brain Research Center, Bar-llan University, RamatGan, 52900, Israel. E-mail: sterned@mail.biu.ac.il.

DOI:10.1523/JNEUROSCI.2426-12.2012

Copyright $\odot 2012$ the authors $\quad 0270-6474 / 12 / 3211241-09 \$ 15.00 / 0$ plaque-burdened neocortex (Knowles et al., 1998, 1999; Le et al., 2001; D'Amore et al., 2003; Lombardo et al., 2003). The increase in neuritic curvature was shown to be correlated with a reduction in synaptic response reliability (Stern et al., 2004). However, the neuronal mechanisms underlying this reduction are unclear because neocortical synaptic inputs have multiple components, both excitatory and inhibitory. It could be that some connectivity patterns are more disrupted by plaques, while others are preferentially spared.

To measure the relationship of plaque distribution to cortical columnar organization, we chose the vibrissae-receptive primary sensory cortex (barrel cortex) as the region of interest. This area, like many other parts of the cortex, is organized in a columnar fashion (Durham and Woolsey, 1977; Woolsey, 1978, 1996; Simons and Woolsey, 1979; Bernardo et al., 1990). However, unlike other sensory areas, the cortical columns are easily identified (Woolsey and Van der Loos, 1970; Axelrad et al., 1976). The discrete barrel-shaped structures located in layer IV of the primary somatosensory cortex, together with their extensions in layer II/III (supragranular layers), and layers V-VI (infragranular layers) represent the functional columns corresponding to the individual rodent vibrissae. These large whiskers on the snout of the animals are arranged in a stereotypical pattern, and the spatial relationship of the pattern is preserved in each of the stages of somatosensory hierarchical processing: the trigeminal nuclei, the thalamus, and the neocortex. Thus the vibrissae maps are preserved much as are the retinotopic maps in the visual system. The barrel columns are segregated from the interbarrel area (septa), in which the neuronal connectivity patterns are different. 
The neurons within the barrels in Layer IV respond primarily to inputs from a single (principal) whisker. The thalamocortical excitatory responses of Layer IV neurons are highly segregated to single barrels (Simons and Carvell, 1989; Goldreich et al., 1999; Laaris et al., 2000; Laaris and Keller, 2002), while the inhibitory input to Layer IV neurons is intracortical and at least partially originate at the septae (McCasland et al., 1991; Petersen and Sakmann, 2001; Sato et al., 2008; Helmstaedter et al., 2009). The receptive fields of these neurons include responses from surround whiskers in addition to the principal whisker. The surround whiskers' inputs to the receptive fields are mediated by intracortical connections. Neurons respond to excitatory and inhibitory inputs from other whiskers to varying degrees, depending on laminar location (Armstrong-James and Fox, 1987; Armstrong-James and Callahan, 1991; Armstrong-James et al., 1991; Brumberg et al., 1999). The segregation of connectivity patterns in barrels and septae in Layer IV provide an anatomical substrate for studying the possible effects of plaques on functional properties of the cortical circuit. Although these specific discontinuities are not present in the human neocortex, the general question of heterogeneous plaque distribution relative to functional cortical columns is crucial to understanding the mechanisms of cognitive disruption in $\mathrm{AD}$ (Knowles et al., 1998, 1999).

To date, neocortical $\mathrm{A} \beta$ plaques distribution have not been well quantified in relation to columnar organization, although it has been hypothesized that plaques disrupt columnar organization (Knowles et al., 1999). The barrel cortex is a convenient system for answering this question: measuring the distribution of plaques in relation to the cortical columns; i.e., whether the plaques are distributed equally between barrels and septae will shed light on the ways in which plaques interact with functional structures within the cortex.

\section{Materials and Methods}

Animals. Six APP/PS1 transgenic mice from the B6C3 strain developed by Jankowsky et al. (2004) were used in this experiment. This strain expresses human presenilin 1 (A246E variant) and a chimeric amyloid precursor protein $\left(\mathrm{APP}_{\text {swe }}\right)$. Amyloid burden and plaque load increase with age, and by 9 months, the hippocampus and cortex of the transgenic mice are largely filled with aggregated peptide (Jankowsky et al., 2004). On the day of the perfusion all six animals used for this study were between 19.5 and 21 months of age. Five female and one male animals were used. Two hemispheres of two different animals, both 20 months of age, were used as a control.

Materials. We measured plaque distribution in relation to barrel boundaries as follows. To identify the barrel structure in layer IV of the somatosensory cortex, cytochrome oxidase staining (Wong-Riley and Welt, 1980) was used on all of the brain sections. Briefly, mounted sections were immersed in a solution containing $300 \mathrm{ml}$ of $0.1 \mathrm{M} \mathrm{PB}, 50 \mathrm{mg}$ of DAB (Sigma-Aldrich), $3 \mathrm{mg}$ of cytochrome $c$ (Sigma-Aldrich) and $11 \mathrm{~g}$ of sucrose (Bio Lab Ltd.). The sections were incubated in the $\mathrm{CO}$ reagent overnight at room temperature. The reaction was stopped when individual barrels were clearly distinguishable from the background, usually after $10-15 \mathrm{~h}$. The sections were washed in $0.1 \mathrm{M}$ PB three times for 20 min at RT and then air-dried.

To visualize the amyloid plaques, the brain sections were processed for Thioflavin-S staining (Nagel et al., 2002). Before starting with the staining procedure, the mounted sections were immersed in 50\% Ethanol for $1 \mathrm{~h}$ at room temperature. Each section was covered by a drop of $0.05 \%$ Thioflavin-S (Sigma-Aldrich) in 50\% Ethanol. In total, the sections were stained for $8 \mathrm{~min}$ in the dark. After the staining, the sections were flooded with two changes of $80 \%$ Ethanol and two changes of distilled water.

To control for possible quenching of the Thioflavin-S fluorescence by $\mathrm{DAB}$, we measured total plaque burden before and following CO staining in one section. While background fluorescence was indeed quenched by

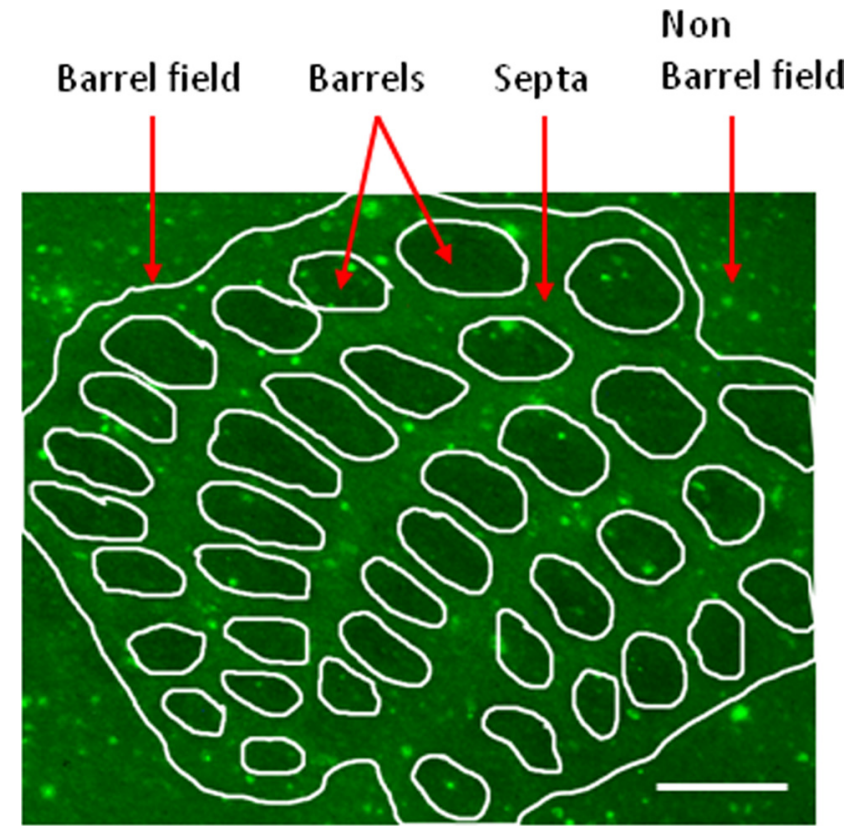

Figure 1. Division of cortical areas for analysis. Cytochrome oxidase-stained tissue was used for separating barrels from septae. Barrels were outlined using Adobe Photoshop, tracing along the border with septae at the points of highest contrast. The barrel field itself was outlined by taking an approximately smooth ellipsoid that included all the barrels. Scale bar, $300 \mu \mathrm{m}$.

the DAB reaction, the plaque burden was not significantly changed before and after Thioflavin-S staining $(t=0.58, \mathrm{df}=14, p=0.57, \mathrm{~ns})$.

Analysis. We defined plaque load as the ratio between the area covered by plaques and the total area in square micrometers. Five different areas were defined for the analysis of the plaque load of granular and supragranular layers: the total area of the image (total), the area of the barrel field (BF), the area of the image surrounding the barrel field (nBF), the sum of the area occupied by barrels within the defined barrel field (barrels), and the total area occupied by the septa region within the defined barrel field (septa). The divisions of the cortical areas are illustrated in Figure 1.

After measuring the individual areas in pixel dimensions, they were converted into square micrometers. Similar methods were used to calculate plaque load. As there are no barrels visible in the supragranular layer, the same five definitions were used to subdivide an image. The so-called barrel field, non-barrel field, barrels, and septa are the exact vertical extension of the matching granular layer. All hypotheses were tested using a $p$ value of 0.05 .

For analysis of spatial relationships among plaques, we used nearest neighbor analysis (Clark and Evans, 1954), which measures the degree of spatial dispersion in the distribution based on the minimum of the interfeature distances. For every brain slice, the average distance between each plaque's center and its nearest neighbor was calculated. This average was compared with the expected value of the average nearest distance, denoted

by $\frac{1}{2} \sqrt{\frac{A}{n}}$, where $A$ is the total plaque area in a given slice and $n$ is number of plaques. This ratio was calculated for each group separately. This measure was chosen because it is relatively insensitive to the number of objects.

\section{Results}

Plaque load was compared between subareas of individual layers and between layers. We found no significant overall differences between the distributions of plaques between left and right hemispheres using a paired $t$ test $(t=-1.95$; df $=5$; ns $)$, and the variability between hemispheres was not significantly different from the variability between mice, allowing us to combine data from both hemispheres in the study.

For the analysis of plaque load occurring in individual barrels and septa it was necessary in some of the images to define the 
Table 1. Mean and SD results for plaque load (PL) in individual brains within five different subareas of the granular and supragranular layer: total area, barrel field, non-barrel field, barrels, and septa

\begin{tabular}{cllllll}
\hline & $\begin{array}{l}\text { Age } \\
\text { (months) }\end{array}$ & $\begin{array}{l}\text { Mean total } \\
\text { PL (\%) }\end{array}$ & $\begin{array}{l}\text { Mean BF } \\
\text { PL (\%) }\end{array}$ & $\begin{array}{l}\text { Mean } \\
\text { non-BF } \\
\text { PL (\%) }\end{array}$ & $\begin{array}{l}\text { Mean } \\
\text { barrels } \\
\text { PL (\%) }\end{array}$ & $\begin{array}{l}\text { Mean } \\
\text { septa } \\
\text { PL (\%) }\end{array}$ \\
\hline Granular layer IV & & & & & & \\
n1 & 21 & 2.12 & 1.49 & 2.66 & 0.5 & 2.24 \\
n2 & 21 & 2.29 & 1.88 & 2.66 & 0.9 & 2.15 \\
n3 & 20.5 & 2.47 & 1.37 & 3.89 & 0.85 & 1.72 \\
n4 & 19.5 & 2.35 & 2.35 & 2.38 & 0.91 & 2.61 \\
n5 & 21 & 2.74 & 1.41 & 4.04 & 0.93 & 0.97 \\
n6 & 21 & 1.15 & 1.08 & 1.76 & 0.4 & 0.74 \\
Mean & $\mathbf{2 0 . 6}$ & $\mathbf{2 . 1 9}$ & $\mathbf{1 . 5 9}$ & $\mathbf{2 . 9 0}$ & $\mathbf{0 . 7 5}$ & $\mathbf{1 . 7 4}$ \\
SD & $\mathbf{0 . 6 0}$ & $\mathbf{1 . 1 9}$ & $\mathbf{0 . 8 4}$ & $\mathbf{1 . 6 7}$ & $\mathbf{0 . 3 1}$ & $\mathbf{1 . 3 9}$ \\
Supragranular & & & & & & \\
layer II/II & & & & & & \\
n1 & 21 & 1.3 & 1.54 & 1.13 & 1.32 & 1.77 \\
n2 & 21 & 0.73 & 0.85 & 0.64 & 0.84 & 0.85 \\
n3 & 20.5 & 0.84 & 1.03 & 0.54 & 0.88 & 1.26 \\
n4 & 19.5 & 0.95 & 1.26 & 0.74 & 1.43 & 1.11 \\
n5 & 21 & 1.04 & 1.16 & 0.98 & 0.81 & 1.58 \\
n6 & 21 & 1.02 & 1.2 & 0.89 & 1.2 & 1.21 \\
Mean & $\mathbf{2 0 . 6}$ & $\mathbf{0 . 9 8}$ & $\mathbf{1 . 1 7}$ & $\mathbf{0 . 8 2}$ & $\mathbf{1 . 0 8}$ & $\mathbf{1 . 2 9}$ \\
SD & $\mathbf{0 . 6 0}$ & $\mathbf{0 . 3 3}$ & $\mathbf{0 . 4 2}$ & $\mathbf{0 . 3 2}$ & $\mathbf{0 . 4 2}$ & $\mathbf{0 . 5 1}$ \\
\hline
\end{tabular}

Statistics were calculated on the two hemispheres together.

barrel field. A smaller area had to be encircled to enable a precise analysis. In some images, barrels did not appear with an accurate outline. For analysis of the barrel field and the non-barrel field, barrels were suitable to be included in the measurement only if they could be sufficiently distinguished. For analysis of the single barrels, each barrel had to be encircled accurately. Therefore, only those barrels with surrounding septa which had clear barrel outlines were included in the respective barrel field, which might underestimate the actual differences between barrel and septal plaques. The area and plaque load of the septa was derived from the difference between the data of barrels and BF. The results of the comparisons in this study are summarized in Table 1.

Figure 2 shows bright-field and fluorescence micrographs of Layer IV barrel cortex. The average plaque load over all areas in the granular layer was $2.19 \pm 1.19 \%$. We first compared plaque load between the barrel field and the non-barrel field in the granular layer. The mean plaque load of the barrel field was $1.59 \pm 0.84 \%$, while the mean plaque load of the non-barrel field was $2.90 \pm 1.67 \%$. The mean plaque load of the matching barrel field at supragranular layer was $1.17 \pm$ $0.42 \%$, while the mean plaque load of the non-barrel field was $0.82 \pm 0.32 \%$. We found a significant main effect for layer $(F=$ 19.94 ; $\mathrm{df}=(1,44) ; p \leq 0.05)$, but not for field (barrel vs nonbarrel; $F=2.87$; $\mathrm{df}=(1,44 ; \mathrm{ns}))$. An interaction was found between layer and field $(F=8.23, \mathrm{df}=1,44 ; p<0.05)$. Post hoc comparisons revealed significant differences between barrel field and non-barrel field in layer IV $(Q=4.65, \mathrm{df}=1,44 ; p<0.05)$ but no differences at layer II/III $(Q=1.26 ; \mathrm{df}=1,44 ; \mathrm{ns})$. Surrounding non-barrel field area and implied plaque burden was calculated by the difference between the total area and the BF area. These results show that the whisker-receptive area is less disrupted by plaques than other cortical areas.

We found that individual plaques can fall in either barrels or septa, with exceptions falling on the boundaries themselves. Examples are shown in Figure 3. Figure 3 illustrates the different types of plaques and their locations that are found in Layer IV barrel cortex. These results show that while the barrel/septa
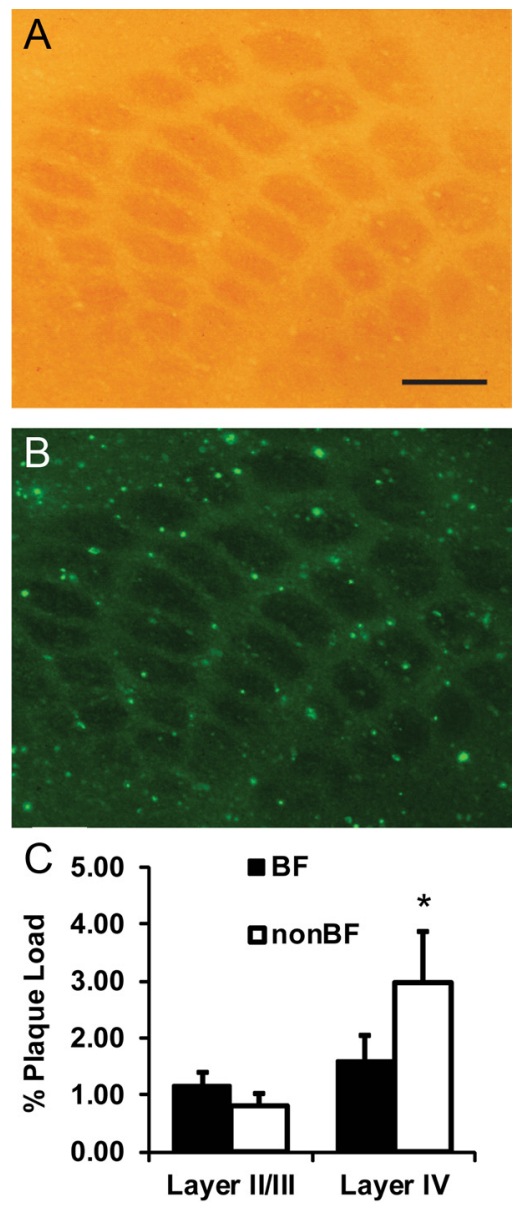

Figure 2. Barrel field in B6C3 APP/PS1 transgenic mice. $A$, Bright-field photograph of layer IV barrel field in a 21-month-old mouse stained for cytochrome oxidase. $\boldsymbol{B}$, Fluorescence (FITC) image of the same area as in A. Tissue is stained with Thioflavine-S. The plaques are seen as bright green spots. C, Comparison of plaque load within and outside the barrel field. The asterisk denotes statistical significance of $p \leq 0.05$. Scale bar, $300 \mu \mathrm{m}$.

boundaries do interact with plaque distribution, plaques can aggregate within both compartments, and even overlap the boundary. Therefore, the boundaries do not serve as an impediment to plaque aggregation.

To analyze whether any differences in plaque distribution between barrels and septae are present in other layers of the cortex, we projected the measured barrel delineation patterns of each brain from Layer IV to the section superficial to that containing the barrel pattern, containing the supragranular layers of the same cortical area. Figure $4 \mathrm{~A}$ illustrates the process by which this was done. The sections were aligned, and the barrel outlines copied from the Layer IV section and pasted on the supragranular layer. Figure $4 B$ illustrates the shift predictor, in which the projected barrel pattern in Layer II/III was shifted by the approximate half-width of a barrel $(\sim 100 \mu \mathrm{m})$ in each of 2 dimensions to control for possible incorrect alignment of the barrels.

To test whether plaques were equally distributed in the barrels and septae within the barrel field, we measured the average plaque load in the barrels and septa in slices containing the granular layer of the cortex, in which barrels were clearly identifiable. An example is shown in Figure 5A. The average plaque load in barrels was $0.75 \pm 0.31 \%$, while that in septa was $1.74 \pm 1.39 \%$.

To test whether these differences were also present between the areas in Layer II/III immediately superficial to those in which we measured in Layer IV, the barrel pattern was marked and 

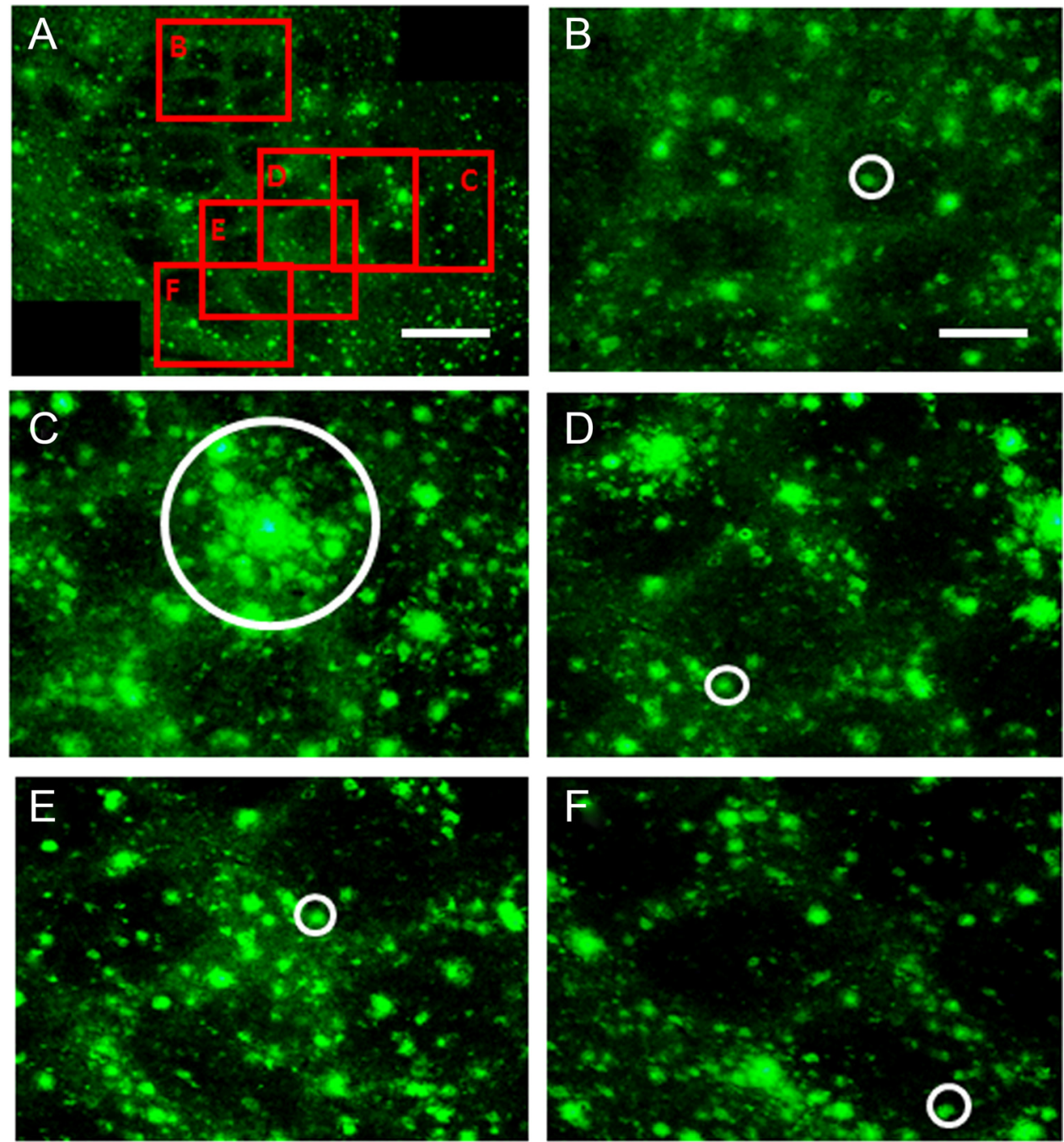

Figure 3. Plaque patterns found in Layer IV barrel cortex. A, An image is shown of Layer IV barrel cortex of a 21-month-old B6C3 APP/PS1 mouse. The red rectangles denote the images shown at higher gain in the other panels of the figure, and the plaques of interest are denoted by the white circles. Scale bar, $300 \mu \mathrm{m}$. $\boldsymbol{B}$, Plaque within barrel. $\boldsymbol{C}$, Large cluster of plaques primarily within septal area. $\boldsymbol{D}, \boldsymbol{E}$, Plaques on boundaries of barrel and septa, primarily within the septa. $\boldsymbol{F}$, Plaque on boundary of barrel and septa, primarily within the barrel. Scale bars $(\boldsymbol{B}-\boldsymbol{F}), 100 \mu \mathrm{m}$.

A

Layer II/III

B

Layer II/III Shifted layer pattern

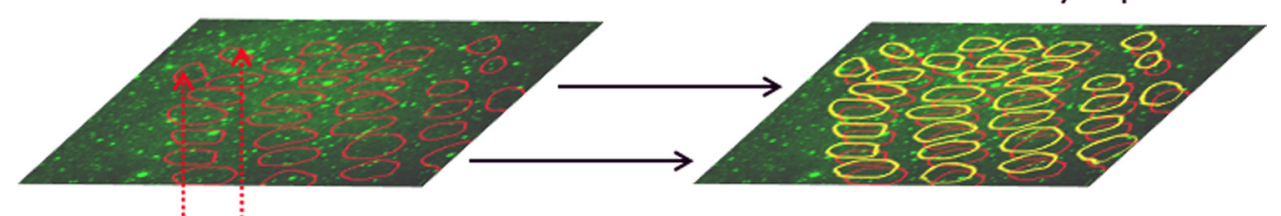

Layer IV

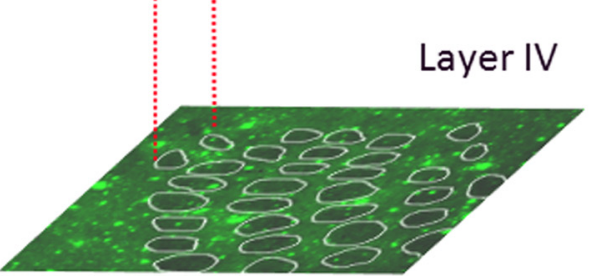

Figure 4. Determination of barrel pattern in Layer II/III and shift predictor. $A$, The barrel edges in Layer IV were detected by contrast, and the boundaries drawn by eye on the edges. This pattern was copied and pasted on the section immediately superior to that containing the barrels, which contained the supragranular layers. The sections were aligned by eye, using slice edges as fiduciary marks. Plaques were then quantified inside and outside the "barrels." $\boldsymbol{B}$, Illustration of the "shift predictor" used to test for artifacts in the alignment. The barrel pattern was superimposed on the section (red pattern) as shown in $\boldsymbol{A}$. The pattern was then shifted by the approximate half-size of a barrel in two dimensions (yellow pattern). Plaques were then quantified inside and outside the "barrels." 

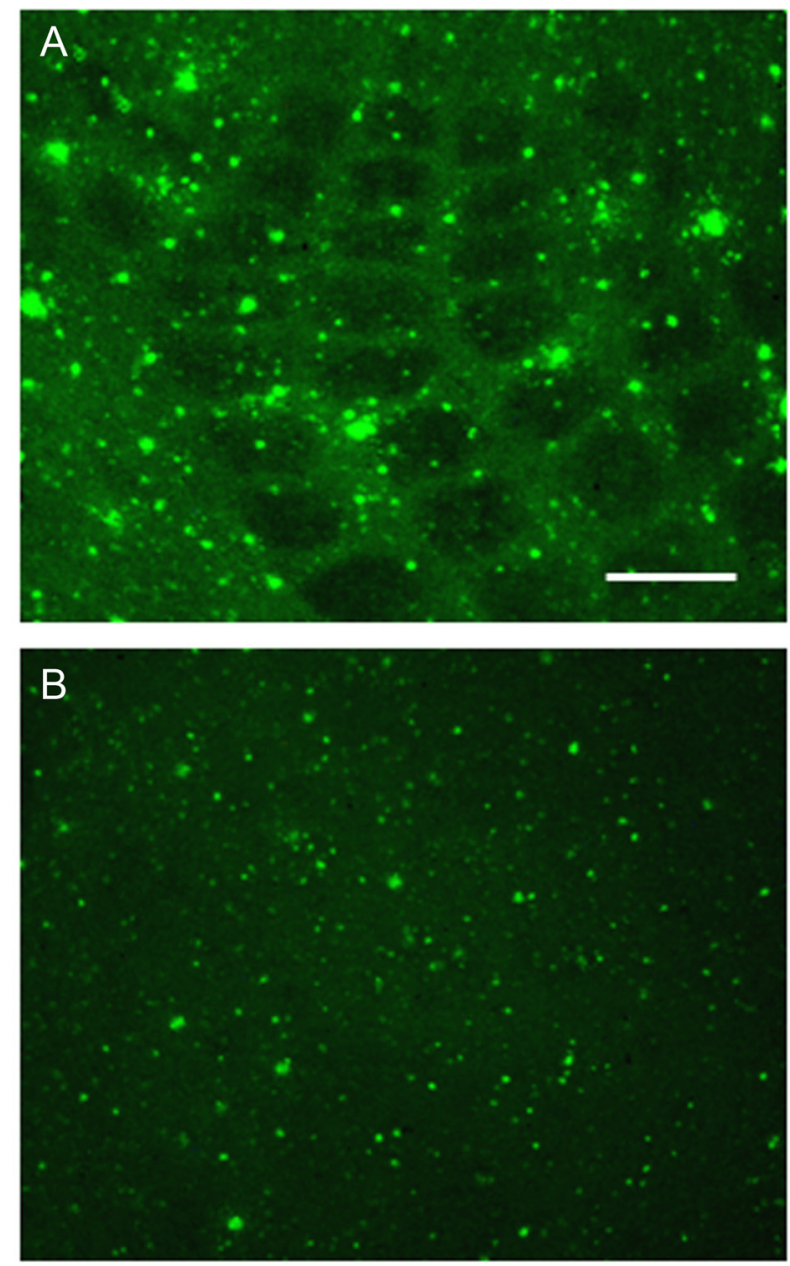

C

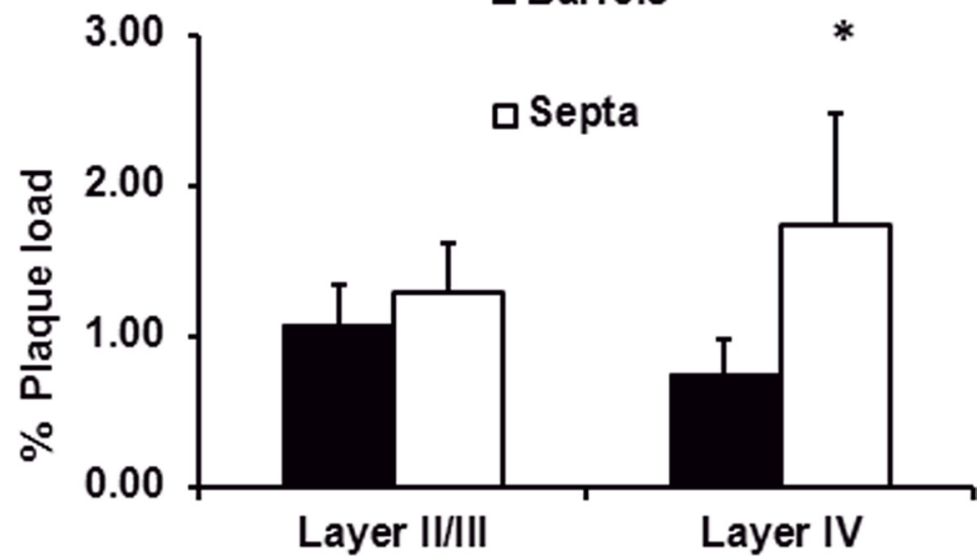

Figure 5. Columnar differences in plaque load in barrel cortex of B6C3 APP/PS1 transgenic mice. $A$, Image of barrel cortex of a 21-month-old B6C3 APP/PS1 mouse stained for cytochrome oxidase and Thioflavine-S. B, Layer II/III barrel cortex in a slice immediately superior to the slice shown in $A$. The barrel pattern in $\boldsymbol{A}$ was traced and superimposed on the image in $\boldsymbol{B}$ to perform the statistical comparison between barrel and septal areas in the supragranular layers. $\boldsymbol{C}$, Significant differences $(p \leq 0.05)$ were found between cortical layers as well as barrel versus septal areas. Post hoc tests revealed that the significant differences were in Layer IV only $\left({ }^{*} p \leq 0.05\right)$. Scale bar, $300 \mu \mathrm{m}$.

transferred to the more superficial section using fiduciary marks to align the sections. An example is shown in Figure $5 B$, which shows the section immediately superficial to that pictured in Figure $3 A$. No barrel patterns are seen. The average plaque load in the Layer II/III barrel continuations was $1.08 \pm 0.42 \%$, while that in the Layer II/III septa continuations was $1.29 \pm 0.51 \%$.
We tested for significant differences in plaque load between barrels and septa and between granular and supragranular layers (see below) using a $2 \times 2$ (barrel/ septa $\times$ granular/supragranular) ANOVA design. The results of this comparison are shown in Figure 5. The main effect of differences between the plaque load in barrels vs septa was significant $(F=6.96$; df $=1,44 ; p \leq 0.05)$, indicating that plaques are distributed in a nonrandom pattern with respect to barrel boundaries. We found no main effect of cortical layer on plaque load ( $F=0.06$; df $=1,44 ; \mathrm{ns})$. We found no layer $\times$ cortical compartment interaction effect $(F=2.89$; $\mathrm{df}=1$, 44; ns). Post hoc analyses showed that the differences in cortical compartments were significant in the granular layer (Tukey post hoc $Q=4.34$; df $=1,44 ; p \leq 0.05$ ), but not in the supragranular layer (Tukey post hoc $Q=0.95$; $\mathrm{df}=1,44$; ns). These results show that plaques are distributed nonrandomly with respect to boundaries in which cortical areas are physically delineated, but not with respect to the columnar continuation of those areas in which the physical delineation is not present.

To control for the possibility that the lack of differences in plaque distribution between Layer II/III barrel and septal continua are an artifact of the incorrect alignment of the horizontal slices, we performed the same analysis as above but with a "shifted" continuation of the barrels, as illustrated in Figure $4 B$. We measured the plaque load in the shifted "barrels" and "septae" using the shift predictor as described above, and repeated the analysis. Our post hoc test of the effect found in the ANOVA revealed that there are no significant differences in plaque load between the shifted barrels and septae in Layer II/III (Tukey HSD; $\mathrm{df}=1,44 ; Q=$ $1.46, \mathrm{~ns})$. This provides evidence that the unequal distribution of plaques between barrel and septae in Layer IV does not continue throughout the cortical column; rather, it is confined to the cortical layer specifically demarcated by the barrel boundaries, despite the fact that individual plaques can be found on both sides of, and even straddling, the barrel boundary.

The above results provide a quantitative description of the relationship of plaques to the columnar organization of the neocortex. It is possible that such a relationship also exists in other sensory areas. However, without the discrete boundary markings provided by the barrels, it was not feasible to measure compartmental differences in other areas. To test for differences in specific patterns of plaques between cortical areas, we used a cluster analysis, which measures the ratio of the average distance between nearest neighbors of plaques to that which would be seen with a 


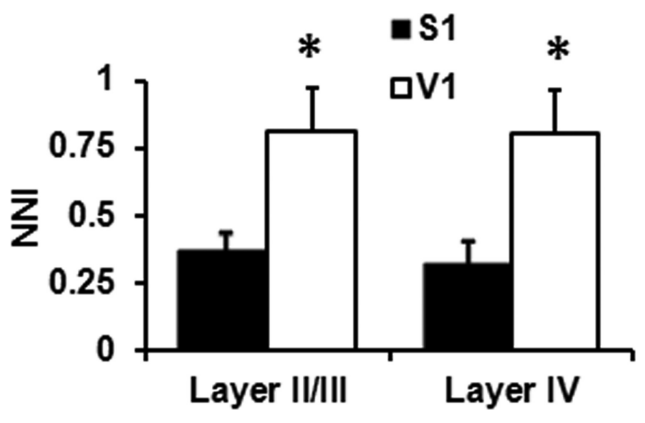

Figure 6. Nearest-neighbor indices for plaques in primary somatosensory and primary visual cortex. Numbers indicate the degree of "clustering" of plaques within that area. A significant main effect was found for sensory area. Plaques in both granular and supragranular layers of $\mathrm{S} 1$ are more clustered than those in the respective layers in V1.

random pattern. According to this cluster analysis, the more closely the points are clustered together, the closer to 0 the nearest-neighbor index (NNI) will be. The closer the NNI gets to 1, the more randomly spaced the points.

Since we expected the clustering pattern of plaques in the primary somatosensory cortex to differ from that of other primary sensory cortex, which lacks the discrete barrel/septa organization, we chose the primary visual cortex (VI) for comparison of plaque clustering to that measured in primary somatosensory cortex. It is generally thought that the columnar organization of the mouse visual cortex is less salient than in other mammals (Hübener, 2003). If plaques are clustered in V1 in a similar fashion to $\mathrm{S} 1$, then we could infer overall "clustering" of plaques in primary sensory cortical areas. On the other hand, if clustering patterns are different between $\mathrm{S} 1$ and V1, then we may conclude that the barrel cortex has a unique spatial arrangement due to its discontinuities.

Nearest neighbor analysis was performed on plaques in supragranular and granular layers of S1 and V1. While the index values of layer IV V1 were closer to 1 (mean $=0.8, \mathrm{SD}=0.17)$, index values of layer IV S1 were closer to 0 (mean $=0.32, \mathrm{SD}=0.07)$, showing a unique spatial arrangement in $\mathrm{S} 1$ : plaques within $\mathrm{S} 1$ are more clustered than those within V1, which tend to be more randomly distributed. Similar results were shown in comparing the supragranular layers of these two sensory areas: index value of layer II/III V1 (mean $=0.8, \mathrm{SD}=0.16$ ) were higher and closer to 1 than this of layer II/III S1 (mean $=0.37, \mathrm{SD}=0.08$ ). These results are shown in the graph in Figure 6.

A two-way ANOVA was computed for that index with a $2 \times$ 2 Sensory Area $\times$ Layer design. We found a significant effect for sensory area $(F=144, \mathrm{df}=1,38 ; p<0.001)$, but not for layer $(F=0.5, \mathrm{df}=1,38 ; p=\mathrm{ns})$. No interaction was found $(F=0.32, \mathrm{df}=1,38 ; p=\mathrm{ns})$. The index values of $\mathrm{V} 1$ layers were closer to 1 (mean $=0.8, \mathrm{SD}=0.16)$, index values of $\mathrm{S} 1$ layers were lower, closer to $0($ mean $=0.34, \mathrm{SD}=0.08)$, showing a unique spatial arrangement in S1; plaques within which are more clustered than those within V1 areas, which tend to be more randomly distributed. The similarity to the effect seen in the supragranular layer shows that the differences between S1 and $\mathrm{V} 1$ are not due only to the cortical discontinuities in the barrel cortex. Although the physical boundaries are not present in the supragranular layer of S1, the plaques tend to cluster within the continuation of the septae in the supragranular layer. While the plaque counts show more plaques in septae than barrels at Layer IV but not II/III, the cluster analysis reveals similar patterns between the layers in each of the two areas.

\section{Discussion}

The results presented in this study show that plaques in the barrel cortex are distributed in a nonrandom pattern. In addition, the plaque aggregation differs between cortical compartment and layer. While the latter has been demonstrated previously (Armstrong et al., 1991; Beach and McGeer, 1992), this is the first time that cortical compartmentalization has been shown to be correlated with neuropathology.

Plaque load is significantly higher in the granular (IV) than in the supragranular (II/III) layer of the somatosensory cortex. In addition, in the granular layer, plaque load is higher in the septa than in the barrels. This difference is not reflected in the supragranular continuation of the functional columns. This has clear implications for the type of neocortical computations that are disrupted in AD. The plaques primarily (but not exclusively) aggregate in septal areas. These areas receive different thalamic and transcallosal information than do the barrels (Keller and White, 1987; White and Keller, 1987), and differential effects of plaques on the two pathways has clear indications for neocortical information processing. For example, the septa are thought to function as intercolumnar areas responsible for computations such as lateral inhibition between adjacent whiskers. This implies that such columnar disruptions may be present in $\mathrm{AD}$ even where clear morphological columnar discontinuities are not readily apparent. The relative randomness of plaques that was found in $\mathrm{V} 1$ of $\mathrm{A} \beta$ mice is compatible with the finding of Beach and McGeer (1992), who found some degree of plaque clustering in layers IVc and V of human striate cortex. In mice, the columnar structure of V1 is much less robust than in humans (Müller et al., 2000; Schuett et al., 2002; Hübener, 2003). The clustering might be related to high columnar organization, which is correlated with the dominance of a specific sensory module. The lack of robust columnar organization in mouse V1 with respect to human V1 is congruent with the relatively low clustering of plaques in mouse cortex found here, as opposed to Beach and McGeer's finding in human cortex. Our findings provide evidence that functional columnar organization is related to plaque clustering patterns within the cortex.

The major implication of this study is that the aggregation of amyloid- $\beta$ is selective; there seems to be a relatively preferred location for the aggregation process. One possible reason for this might be that the septa have a biophysical substrate that acts as a preferential site for amyloid- $\beta$ aggregation. The pattern of barrel/ septal organization is laid out in the early stage of brain development by boundaries comprised of glial cells and glycoconjugates. These boundaries are widespread during CNS pattern formation and disappear following synaptic stabilization (Steindler et al., 1990, 1998; Steindler, 1993). However, these molecules become active in cases of brain injury and brain pathology (Laywell and Steindler, 1991). Brain injuries are known to be a major risk factor for Alzheimer's and other neurodegenerative diseases (Herrup; DeKosky et al., 2007; Adibhatla and Hatcher, 2008; Pluta and Amek, 2008; Härtig et al., 2009). It may be that plaques could be considered as a type of brain injury, disrupting the cytoarchitecture (Knowles et al., 1998; Knowles et al., 1999), being generally neurotoxic (Urbanc et al., 2002; Velez-Pardo et al., 2004; Floden et al., 2005; Spires et al., 2005; Naylor et al., 2008a,b), and causing a general immune response (Eikelenboom et al., 2006; Garcia-Alloza et al., 2007; Koenigsknecht-Talboo et al., 2008; Meyer-Luehmann et al., 2008; Combs, 2009; Mundt et al., 2009; Salmina, 2009).

Although boundaries such as those observed in the rodent barrel field are not observed in human brain, this may serve as a model for other boundaries which define the cortical cytoarchi- 


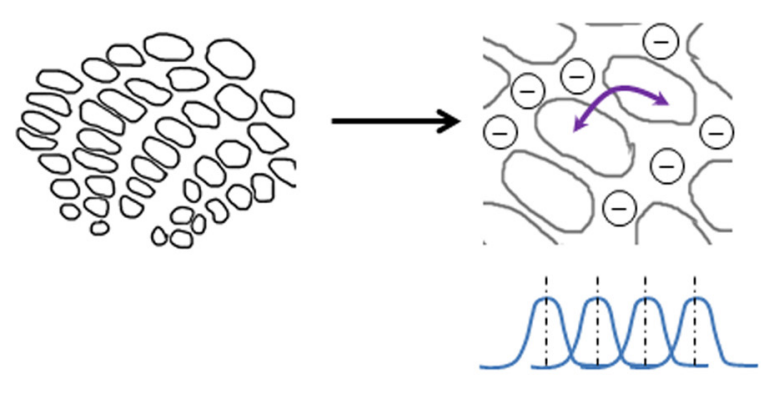

Normal receptive

fields

\section{B}
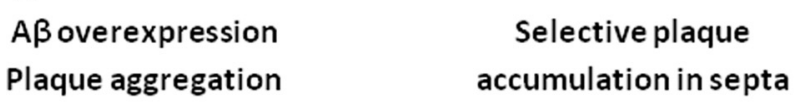

Reduced lateral inhibition
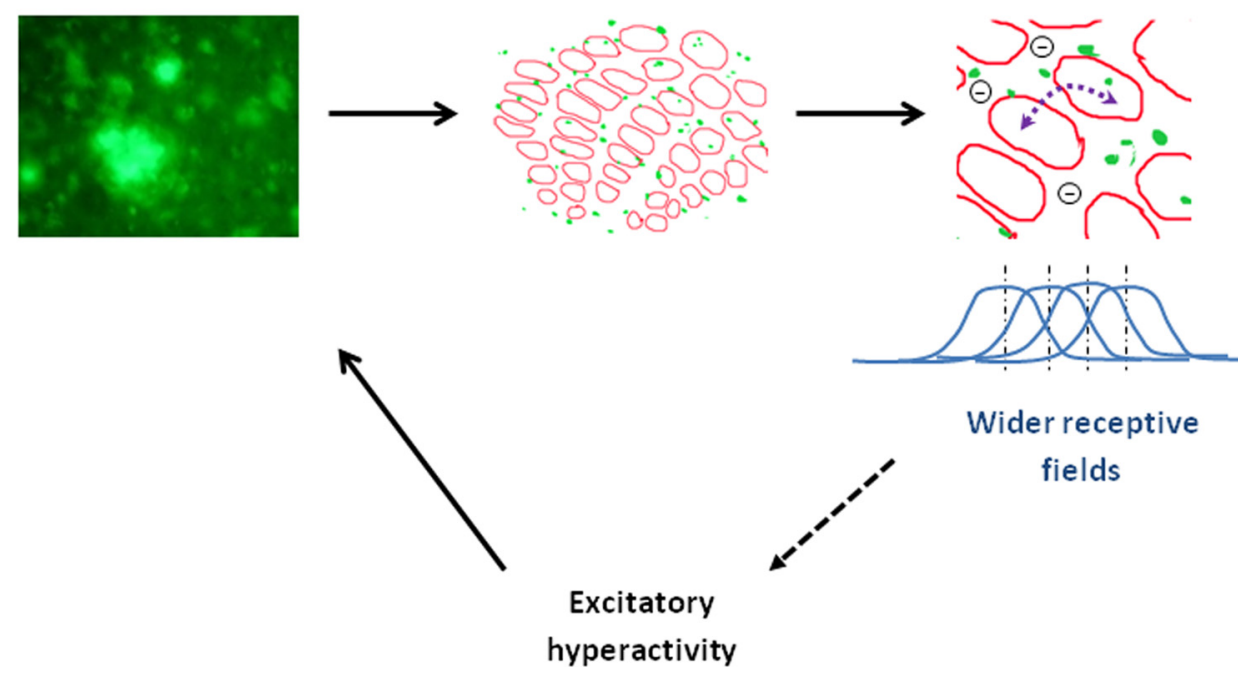

Figure 7. Proposed model for interaction between selective plaque accumulation and changes in Layer IV somatosensory receptive fields in amyloid- $\beta$ mouse model. $\boldsymbol{A}$, In normal cortex, inhibitory interneurons in the septae mediate the receptive fields of the neurons in the barrels by lateral inhibition of inputs from neighboring whiskers (symbolized by the dashed-circle symbols and thick purple arrows). $\boldsymbol{B}$, Plaques accumulate in the septae and reduce the activity of the inhibitory neurons. This reduces the amount of lateral inhibition between whisker inputs, resulting in broadening the receptive fields of the barrel neurons, and thus reducing response specificity in these cells. In addition, the reduction of inhibitory neuronal activity in the septae may result in the increased production of amyloid- $\beta$ in these areas, contributing to the creation of more plaques, in an excitatory feedback loop.

tecture. In any case, this study shows that the physical discontinuities caused by the barrels do influence the distribution of the neuropathology, even in areas where the discontinuities themselves are not present, such as Layers II/III. This has clear implications for the functional effects of plaques on cortical organization. For example, communication between cortical columns in this system is more likely to be affected (and to greater degree) than communication within columns.

One possible way in which intracortical information processing may be specifically affected by differential plaque distribution patterns is the balance between specific excitatory and inhibitory circuits. There is evidence that neuronal activity modulates the secretion of amyloid- $\beta$ (Kamenetz et al., 2003; Cirrito et al., 2005, 2008). In these lines of experiments, it was suggested that neuronal activity may accelerate $A \beta$ concentration. When barrel cortex neurons are deprived of sensory afferent information, plaque formation is slowed (Bero et al., 2011). In addition, clusters of hyperactive neurons have been found near plaques (where presumably concentrations of soluble amyloid- $\beta$ are high), and it has been suggested that this hyperactivity is due to a relative decrease in synaptic inhibition (Busche et al., 2008). These studies suggest that inhibitory synaptic input is selectively vulnerable to plaque aggregation. This may in turn compensate for the reduction in synaptic reliability observed in the presence of $A \beta$ plaques (Stern et al., 2004). This reduction was hypothesized to be caused by plaque-induced physical distortion of afferent fibers, which would in turn cause a temporal desynchronization of synaptic inputs. Such a desynchronization is sufficient to cause greater reduced average synaptic responses, and might cause increased within-state fluctuations. We propose that the results of our study have clear implications for the functional effects of plaques on cortical activity. Figure 7 illustrates a model of such possible effect. In normal barrel cortex, inhibitory synaptic inputs play a number of roles in shaping the sensory responses (Fig. 7A). One such role is lateral inhibition between adjacent whisker inputs, which occurs in Layer IV (Sato et al., 2008). The 
lateral inhibition is mediated by inhibitory interneurons in the septae between the barrels, where, as shown in our study, plaque aggregation is selectively prevalent. A major function of lateral inhibition is sharpening the sensory tuning properties of the thalamic-input recipient neurons in the barrels (Fig. 7, illustrated by the Gaussian "tuning curves"). This sharpening could play a major role in behavior, increasing the ability to detect edges and discriminate textures. In the $\mathrm{AD}$ model (Fig. $7 B$ ), accumulation of plaques in the septa cause a reduction in the lateral inhibition between barrels, as plaques have been shown to reduce inhibition in other cortical areas (Busche et al., 2008). We propose that this would cause a broadening of tuning of the whisker-evoked Layer IV field potential of the barrel cortex, which would be accompanied by a reduction in the behavioral ability of the mice to distinguish between surfaces in a somatosensory discrimination task. Specifically, it would reduce the ability to distinguish high spatial frequencies and as a result, reduce the animal's ability for edge detection.

The reduced inhibition in the septal areas could in turn lead to increased excitatory activity of the neurons in these areas. Increased activity has been shown to mediate release of amyloid- $\beta$ by neurons (Kamenetz et al., 2003), which could lead to further plaque aggregation in a positive feedback loop. This hypothesis could be tested electrophysiologically by intracellular and extracellular recordings, as well as longitudinal studies of plaque aggregation over time, as our model would predict supralinear accumulation of amyloid- $\beta$ and plaques in these areas.

In general, long-range neuritic trajectories communicate between functional columns such as those seen in barrel cortex and hence are related to global processing. It may be that cognitive tasks involving global processing of information would be expected to be more affected than cognitive tasks involving local processing in $\mathrm{AD}$. Although we cannot directly measure such columnar disruption in humans, our results in mouse models do allow us to make experimental predictions. The preceding example of global versus local information processing is one such; presenting such a test measuring performance on both to $\mathrm{AD}$ patients is a relatively straightforward process.

This is the first time to our knowledge that plaque distribution has been quantified in relation to cortical compartmentalization. The difference in relative proportion of plaques within barrels and septae of S1 does not extrapolate to layers and areas in which barrel boundaries are not found. However, the pattern of plaque distribution, as measured by clustering, does extrapolate to the supragranular layer of S1, where it differs significantly from that in V1. We conclude that plaque distribution within cortical areas is partially determined by constraints such as boundaries, even if these boundaries are localized to more specific subareas. This may be due to a greater tendency of specific neurons or spatial arrangements of neurons to serve as a substrate for plaque aggregation. This would imply that there are at least two separate mechanisms involved in the distribution of plaques in the neocortex: the concentration of amyloid- $\beta$ determines the amount of plaque load in a given area, while the local architectonics of the cortex determines the distribution pattern among areas.

\section{References}

Adibhatla RM, Hatcher JF (2008) Altered lipid metabolism in brain injury and disorders. Subcell Biochem 49:241-268.

Armstrong RA, Myers D, Smith CU, Cairns N, Luthert PJ (1991) Alzheimer's disease: the relationship between the density of senile plaques, neurofibrillary tangles and A4 protein in human patients. Neurosci Lett 123:141-143.

Armstrong-James M, Callahan CA (1991) Thalamo-cortical processing of vibrissal information in the rat. II. spatiotemporal convergence in the thalamic ventroposterior medial nucleus (VPm) and its relevance to generation of receptive fields of S1 cortical "barrel" neurones. J Comp Neurol 303:211-224.

Armstrong-James M, Fox K (1987) Spatiotemporal convergence and divergence in the rat S1 "barrel" cortex. J Comp Neurol 263:265-281.

Armstrong-James M, Callahan CA, Friedman MA (1991) Thalamo-cortical processing of vibrissal information in the rat. I. Intracortical origins of surround but not centre-receptive fields of layer IV neurones in the rat S1 barrel field cortex. J Comp Neurol 303:193-210.

Axelrad H, Verley R, Farkas E (1976) Responses evoked in mouse and rat SI cortex by vibrissa stimulation. Neurosci Lett 3:265-274.

Beach TG, McGeer EG (1992) Senile plaques, amyloid beta-protein, and acetylcholinesterase fibres: laminar distributions in Alzheimer's disease striate cortex. Acta Neuropathol 83:292-299.

Bernardo KL, McCasland JS, Woolsey TA, Strominger RN (1990) Local intra- and interlaminar connections in mouse barrel cortex. J Comp Neurol 291:231-255.

Bero AW, Yan P, Roh JH, Cirrito JR, Stewart FR, Raichle ME, Lee JM, Holtzman DM (2011) Neuronal activity regulates the regional vulnerability to amyloid-beta deposition. Nat Neurosci 14:750-756.

Brumberg JC, Pinto DJ, Simons DJ (1999) Cortical columnar processing in the rat whisker-to-barrel system. J Neurophysiol 82:1808-1817.

Busche MA, Eichhoff G, Adelsberger H, Abramowski D, Wiederhold $\mathrm{KH}$, Haass C, Staufenbiel M, Konnerth A, Garaschuk O (2008) Clusters of hyperactive neurons near amyloid plaques in a mouse model of Alzheimer's disease. Science 321:1686-1689.

Cirrito JR, Yamada KA, Finn MB, Sloviter RS, Bales KR, May PC, Schoepp DD, Paul SM, Mennerick S, Holtzman DM (2005) Synaptic activity regulates interstitial fluid amyloid-beta levels in vivo. Neuron 48:913-922.

Cirrito JR, Kang JE, Lee J, Stewart FR, Verges DK, Silverio LM, Bu G, Mennerick S, Holtzman DM (2008) Endocytosis is required for synaptic activity-dependent release of amyloid-beta in vivo. Neuron 58:42-51.

Clark PJ, Evans FC (1954) Distance to nearest neighbor as a measure of spatial relationships in populations. Ecology 35:445-453.

Combs CK (2009) Inflammation and microglia actions in Alzheimer's disease. J Neuroimmune Pharmacol 4:380-388.

D’Amore JD, Kajdasz ST, McLellan ME, Bacskai BJ, Stern EA, Hyman BT (2003) In vivo multiphoton imaging of a transgenic mouse model of Alzheimer disease reveals marked thioflavine-S-associated alterations in neurite trajectories. J Neuropathol Exp Neurol 62:137-145.

DeKosky ST, Abrahamson EE, Ciallella JR, Paljug WR, Wisniewski SR, Clark RS, Ikonomovic MD (2007) Association of increased cortical soluble abeta 42 levels with diffuse plaques after severe brain injury in humans. Arch Neurol 64:541-544.

Durham D, Woolsey TA (1977) Barrels and columnar cortical organization: evidence from 2-deoxyglucose (2-DG) experiments. Brain Res 137:169-174

Eikelenboom P, Veerhuis R, Scheper W, Rozemuller AJ, van Gool WA, Hoozemans JJ (2006) The significance of neuroinflammation in understanding Alzheimer's disease. J Neural Transm 113:1685-1695.

Floden AM, Li S, Combs CK (2005) Beta-amyloid-stimulated microglia induce neuron death via synergistic stimulation of tumor necrosis factor alpha and NMDA receptors. J Neurosci 25:2566-2575.

Garcia-Alloza M, Ferrara BJ, Dodwell SA, Hickey GA, Hyman BT, Bacskai BJ (2007) A limited role for microglia in antibody mediated plaque clearance in APP mice. Neurobiol Dis 28:286-292.

Goldreich D, Kyriazi HT, Simons DJ (1999) Functional independence of layer IV barrels in rodent somatosensory cortex. J Neurophysiol 82:1311-1316.

Härtig W, Reichenbach A, Voigt C, Boltze J, Bulavina L, Schuhmann MU, Seeger J, Schusser GF, Freytag C, Grosche J (2009) Triple fluorescence labelling of neuronal, glial and vascular markers revealing pathological alterations in various animal models. J Chem Neuroanat 37:128-138.

Helmstaedter M, Sakmann B, Feldmeyer D (2009) Neuronal correlates of local, lateral, and translaminar inhibition with reference to cortical columns. Cereb Cortex 19:926-937.

Herrup K (2010) Reimagining Alzheimer's disease-an age-based hypothesis. J Neurosci 30:16755-16762.

Hsiao K, Chapman P, Nilsen S, Eckman C, Harigaya Y, Younkin S, Yang F, Cole G (1996) Correlative memory deficits, Abeta elevation, and amyloid plaques in transgenic mice. Science 274:99-102. 
Hübener M (2003) Mouse visual cortex. Curr Opin Neurobiol 13:413-420.

Hyman BT (2006) The natural history of Alzheimer disease dissected through multiphoton imaging of transgenic mice. Alzheimer Dis Assoc Disord 20:206-209.

Jankowsky JL, Slunt HH, Gonzales V, Jenkins NA, Copeland NG, Borchelt DR (2004) APP processing and amyloid deposition in mice haploinsufficient for presenilin 1. Neurobiol Aging 25:885-892.

Kamenetz F, Tomita T, Hsieh H, Seabrook G, Borchelt D, Iwatsubo T, Sisodia S, Malinow R (2003) APP processing and synaptic function. Neuron 37:925-937.

Keller A, White EL (1987) Synaptic organization of GABAergic neurons in the mouse SmI cortex. J Comp Neurol 262:1-12.

Knowles RB, Gomez-Isla T, Hyman BT (1998) Abeta associated neuropil changes: correlation with neuronal loss and dementia. J Neuropathol Exp Neurol 57:1122-1130.

Knowles RB, Wyart C, Buldyrev SV, Cruz L, Urbanc B, Hasselmo ME, Stanley HE, Hyman BT (1999) Plaque-induced neurite abnormalities: implications for disruption of neural networks in Alzheimer's disease. Proc Natl Acad Sci U S A 96:5274-5279.

Koenigsknecht-Talboo J, Meyer-Luehmann M, Parsadanian M, GarciaAlloza M, Finn MB, Hyman BT, Bacskai BJ, Holtzman DM (2008) Rapid microglial response around amyloid pathology after systemic antiAbeta antibody administration in PDAPP mice. J Neurosci 28:1415614164.

Laaris N, Keller A (2002) Functional independence of layer IV barrels. J Neurophysiol 87:1028-1034.

Laaris N, Carlson GC, Keller A (2000) Thalamic-evoked synaptic interactions in barrel cortex revealed by optical imaging. J Neurosci 20: $1529-1537$.

Laywell ED, Steindler DA (1991) Boundaries and wounds, glia and glycoconjugates. Cellular and molecular analyses of developmental partitions and adult brain lesions. Ann N Y Acad Sci 633:122-141.

Le R, Cruz L, Urbanc B, Knowles RB, Hsiao-Ashe K, Duff K, Irizarry MC, Stanley HE, Hyman BT (2001) Plaque-induced abnormalities in neurite geometry in transgenic models of Alzheimer disease: implications for neural system disruption. J Neuropathol Exp Neurol 60:753-758.

Lombardo JA, Stern EA, McLellan ME, Kajdasz ST, Hickey GA, Bacskai BJ, Hyman BT (2003) Amyloid-beta antibody treatment leads to rapid normalization of plaque-induced neuritic alterations. J Neurosci 23:1087910883.

Mattson MP (2004) Pathways towards and away from Alzheimer's disease. Nature 430:631-639.

McCasland JS, Carvell GE, Simons DJ, Woolsey TA (1991) Functional asymmetries in the rodent barrel cortex. Somatosens Mot Res 8:111-116.

Mehraein P, Yamada M, Tarnowska-Dziduszko E (1975) Quantitative study on dendrites and dendritic spines in Alzheimer's disease and senile dementia. Adv Neurol 12:453-458.

Meyer-Luehmann M, Spires-Jones TL, Prada C, Garcia-Alloza M, de Calignon A, Rozkalne A, Koenigsknecht-Talboo J, Holtzman DM, Bacskai BJ, Hyman BT (2008) Rapid appearance and local toxicity of amyloid-beta plaques in a mouse model of Alzheimer's disease. Nature 451:720-724.

Müller T, Stetter M, Hübener M, Sengpiel F, Bonhoeffer T, Gödecke I, Chapman B, Löwel S, Obermayer K (2000) An analysis of orientation and ocular dominance patterns in the visual cortex of cats and ferrets. Neural Comput 12:2573-2595.

Mundt AP, Winter C, Mueller S, Wuerfel J, Tysiak E, Schnorr J, Taupitz M, Heinz A, Juckel G (2009) Targeting activated microglia in Alzheimer's pathology by intraventricular delivery of a phagocytosable MRI contrast agent in APP23 transgenic mice. Neuroimage 46:367-372.

Nagel G, Ollig D, Fuhrmann M, Kateriya S, Musti AM, Bamberg E, Hegemann P (2002) Channelrhodopsin-1: a light-gated proton channel in green algae. Science 296:2395-2398.

Naylor R, Hill AF, Barnham KJ (2008a) Is covalently crosslinked Abeta responsible for synaptotoxicity in Alzheimer's disease? Curr Alzheimer Res 5:533-539.

Naylor R, Hill AF, Barnham KJ (2008b) Neurotoxicity in Alzheimer's disease: is covalently crosslinked A beta responsible? Eur Biophys J 37: 265-268.

Palop JJ, Chin J, Mucke L (2006) A network dysfunction perspective on neurodegenerative diseases. Nature 443:768-773.
Petersen CC, Sakmann B (2001) Functionally independent columns of rat somatosensory barrel cortex revealed with voltage-sensitive dye imaging. J Neurosci 21:8435-8446.

Pluta R, Amek MU (2008) Brain ischemia and ischemic blood-brain barrier as etiological factors in sporadic Alzheimer's disease. Neuropsychiatr Dis Treat 4:855-864.

Salmina AB (2009) Neuron-glia interactions as therapeutic targets in neurodegeneration. J Alzheimers Dis 16:485-502.

Sato H, Shimanuki Y, Saito M, Toyoda H, Nokubi T, Maeda Y, Yamamoto T, Kang Y (2008) Differential columnar processing in local circuits of barrel and insular cortices. J Neurosci 28:3076-3089.

Schuett S, Bonhoeffer T, Hübener M (2002) Mapping retinotopic structure in mouse visual cortex with optical imaging. J Neurosci 22:6549-6559.

Selkoe DJ (2001) Alzheimer's disease: genes, proteins, and therapy. Physiol Rev 81:741-766.

Selkoe DJ (2002) Alzheimer's disease is a synaptic failure. Science 298: 789-791.

Simons DJ, Carvell GE (1989) Thalamocortical response transformation in the rat vibrissa/barrel system. J Neurophysiol 61:311-330.

Simons DJ, Woolsey TA (1979) Functional organization in mouse barrel cortex. Brain Res 165:327-332.

Spires TL, Hyman BT (2004) Neuronal structure is altered by amyloid plaques. Rev Neurosci 15:267-278.

Spires TL, Meyer-Luehmann M, Stern EA, McLean PJ, Skoch J, Nguyen PT, Bacskai BJ, Hyman BT (2005) Dendritic spine abnormalities in amyloid precursor protein transgenic mice demonstrated by gene transfer and intravital multiphoton microscopy. J Neurosci 25:7278-7287.

Spires-Jones TL, Meyer-Luehmann M, Osetek JD, Jones PB, Stern EA, Bacskai BJ, Hyman BT (2007) Impaired spine stability underlies plaquerelated spine loss in an Alzheimer's disease mouse model. Am J Pathol 171:1304-1311.

Steindler DA (1993) Glial boundaries in the developing nervous system. Annu Rev Neurosci 16:445-470.

Steindler DA, O'Brien TF, Laywell E, Harrington K, Faissner A, Schachner M (1990) Boundaries during normal and abnormal brain development: in vivo and in vitro studies of glia and glycoconjugates. Exp Neurol 109:35-56.

Steindler DA, Kukekov VG, Thomas LB, Fillmore H, Suslov O, Scheffler B, O’Brien TF, Kusakabe M, Laywell ED (1998) Boundary molecules during brain development, injury, and persistent neurogenesis-in vivo and in vitro studies. Prog Brain Res 117:179-196.

Stern EA, Bacskai BJ, Hickey GA, Attenello FJ, Lombardo JA, Hyman BT (2004) Cortical synaptic integration in vivo is disrupted by amyloid-beta plaques. J Neurosci 24:4535-4540.

Tsai J, Grutzendler J, Duff K, Gan WB (2004) Fibrillar amyloid deposition leads to local synaptic abnormalities and breakage of neuronal branches. Nat Neurosci 7:1181-1183.

Urbanc B, Cruz L, Le R, Sanders J, Ashe KH, Duff K, Stanley HE, Irizarry MC, Hyman BT (2002) Neurotoxic effects of thioflavin S-positive amyloid deposits in transgenic mice and Alzheimer's disease. Proc Natl Acad Sci U S A 99:13990-13995.

Velez-Pardo C, Arellano JI, Cardona-Gomez P, Jimenez Del Rio M, Lopera F, De Felipe J (2004) CA1 hippocampal neuronal loss in familial Alzheimer's disease presenilin-1 E280A mutation is related to epilepsy. Epilepsia 45:751-756

White EL, Keller A (1987) Intrinsic circuitry involving the local axon collaterals of corticothalamic projection cells in mouse SmI cortex. J Comp Neurol 262:13-26.

Wong-Riley MT, Welt C (1980) Histochemical changes in cytochrome oxidase of cortical barrels after vibrissal removal in neonatal and adult mice. Proc Natl Acad Sci U S A 77:2333-2337.

Woolsey TA (1978) Some anatomical bases of cortical somatotopic organization. Brain Behav Evol 15:325-371.

Woolsey TA (1996) Barrels: 25 years later. Somatosens Mot Res 13:181-186.

Woolsey TA, Van der Loos H (1970) The structural organization of layer IV in the somatosensory region (SI) of mouse cerebral cortex. The description of a cortical field composed of discrete cytoarchitectonic units. Brain Res 17:205-242. 